\title{
EXCIMER PUMPED DYE MIXTURES FOR THE WAVELENGTH REGION 340-380 nm
}

\author{
D. A. LANDIS, D. J. SCHNEIDER and C. J. SELISKAR $\dagger$ \\ Chemistry Department, University of Cincinnati, \\ Cincinnati, Ohio 45221-0172
}

(Received 15 November, 1990; in final form 29 January, 1991)

\begin{abstract}
Two binary mixtures of ultraviolet laser dyes in $p$-dioxane solution are described. The tuning curve of each mixture spans more than $30 \mathrm{~nm}$ of useful tuning range in the $340-380 \mathrm{~nm}$ wavelength region. Both mixtures are robust with half-lives comparable to, or in excess of, associated single dye values. These mixtures degrade symmetrically with dye laser operation, making them useful throughout the entire life of the mixture. A simple model for the mixture lasing, based on independent fluorescences of the component dyes, is able to semi-quantitatively match the tuning curves of the dye mixtures. The results presented should be easily extendable to other dye mixtures.
\end{abstract}

KEY WORDS: Laser dye mixture, ultraviolet laser dye.

\section{INTRODUCTION}

Tunable laser radiation in the near ultraviolet region, $330-390 \mathrm{~nm}$, is directly available without frequency doubling using organic dyes $(1,2)$ pumped by the $308 \mathrm{~nm}$ excimer laser. As such, an important region of the spectrum of many chemicals is able to be interrogated piecewise in roughly $15-20 \mathrm{~nm}$ segments of tunable dye laser light. Although there have been several recent additions to the selection of near ultraviolet laser dyes (2), the narrow tunability of these dyes remains an inconvenience in many spectroscopic studies. In an effort aimed at designing and testing new ultraviolet laser dyes, we developed two binary laser dye mixtures which appear to be generally useful. This communication briefly describes these dye mixtures and some of the associated physical properties.

\section{EXPERIMENTAL DETAILS}

The laser system, the signal acquisition equipment and the experimental methods used in these studies have been described in detail before (2). Very briefly, the laser system consisted of a Lambda Physik EMG $201 \mathrm{MSC} \mathrm{XeCl}$ excimer laser and FL 3002E dye laser pumped with half of the excimer laser output. Dye circulation was done with the Lambda Physik high-speed flow cells and small $(200 \mathrm{ml})$ dye

$\dagger$ To whom correspondence is to be sent. 
circulators for reasons described in our previous paper on the ultraviolet dyes (2). Fluorescence spectra of dilute samples (absorbance at $308 \mathrm{~nm}$ less than 0.10 ) were taken on a Perkin Elmer LS-5 fluorimeter and have not been corrected for instrumental response. All fluorescence spectra were measured using $308 \mathrm{~nm}$ as the excitation wavelength.

$p$-Terphenyl (PTP), Exalite 348 (TTPTP), DMQ, and $p$-quaterphenyl (PQP) laser dyes were obtained from Exciton, Inc. The solvent $p$-dioxane, freshly opened, was used for all studies described and was purchased from Fisher Scientific as an Analytical Reagent.

\section{RESULTS AND DISCUSSION}

Two dye mixtures have been devised and have been found to perform well under conditions equivalent to those for single dye usage. These mixtures are of the dyes PTP and DMQ, and of TTPTP and PQP.

\section{The PTP/DMQ Mixture}

For the sake of reference, the tuning curves (optical gain versus wavelength curves) and the fluorescence spectra for PTP and for DMQ are shown in Figures 1a and 1d.

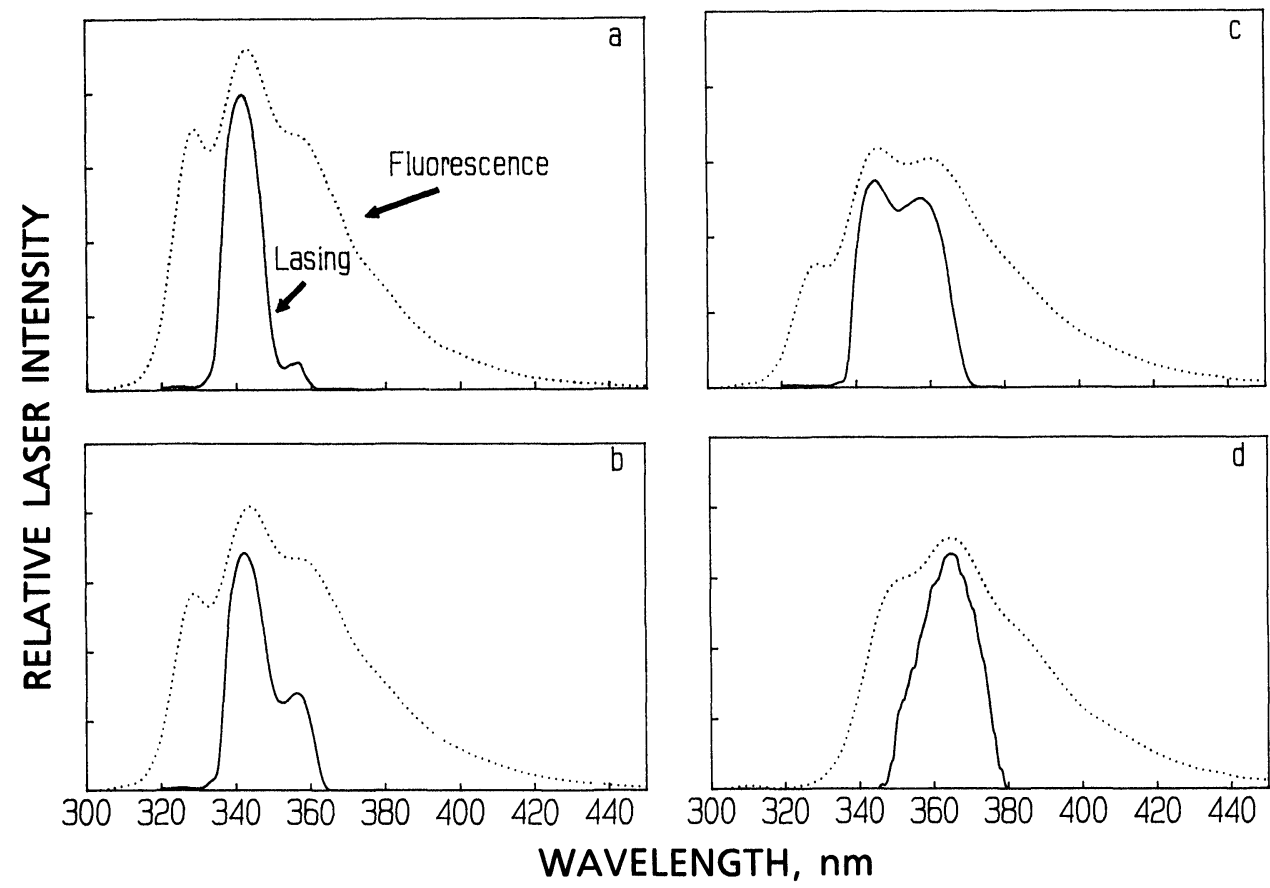

Figure 1 The computed fluorescence spectra (dotted lines) and laser tuning curves (solid lines) for the PTP/DMQ dye mixture. All the tuning curves are correctly vertically scaled with respect to each other. Panel 1a: PTP only; Panel 1b: [PTP] $=1.4 \times 10^{-3} \mathrm{M}, \quad[\mathrm{DMQ}]=9.0 \times 10^{-5} \mathrm{M}$; Panel 1c: $[\mathrm{PTP}]=1.1 \times 10^{-3} \mathrm{M},[\mathrm{DMQ}]=0.31 \times 10^{-3} \mathrm{M} ;$ Panel 1d: DMQ only. 
Related dye parameters are summarized in Table 1 . The individual dyes lase with their maxima being some $20 \mathrm{~nm}$ apart. Roughly speaking, the maximum in each tuning curve represents one vibronic component of the corresponding fluorescence. The tuning curve for PTP shows a small peak at $358 \mathrm{~nm}$ which arises from the third vibronic component in its fluorescence spectrum. The unusually large breadth of the DMQ tuning curve (2) arises from the weak lasing of the first vibronic component of the fluorescence at $352 \mathrm{~nm}$.

The concentration dependence of the dye mixture was determined in two different mixing experiments as follows:

Table 1 Physical characteristics of dyes and dye mixtures

\begin{tabular}{|c|c|c|c|c|c|c|}
\hline \multirow[t]{2}{*}{ Dye } & \multirow{2}{*}{$\begin{array}{l}\text { Optimum } \\
\text { concentration } \\
\text { (mol/l) }\end{array}$} & \multirow{2}{*}{$\begin{array}{l}\text { Lasing } \\
\text { maximum } \\
(\mathrm{nm})\end{array}$} & \multicolumn{2}{|c|}{ Tuning range $(\mathrm{nm})$} & \multirow{2}{*}{$\begin{array}{l}E_{1 / 2} \\
(k J / l)\end{array}$} & \multirow{2}{*}{$\begin{array}{l}\text { Conversion } \\
\text { efficiency } \\
(\%)\end{array}$} \\
\hline & & & $F W H M$ & Full & & \\
\hline PTP & $1.5 \times 10^{-3}$ & 342 & $337-349$ & 329-349 & 640 & 12 \\
\hline ТTPTP & $1.4 \times 10^{-3}$ & 348 & $340-356$ & $333-359$ & 930 & 10 \\
\hline DMQ & $7.2 \times 10^{-4}$ & 365 & $353-374$ & $347-378$ & 960 & 10 \\
\hline PQP & $3.4 \times 10^{-4}$ & $352,368^{\mathrm{b}}$ & $361-376$ & $348-382^{2}$ & 1000 & 14 \\
\hline PTP/DMQ & $1.1 \times 10^{-3} / 0.31 \times 10^{-3}$ & 344,356 & $339-366$ & $337-371$ & 980 & 9 \\
\hline TTPTP/PQP & $6.8 \times 10^{-4} / 3.5 \times 10^{-4}$ & 352,367 & $347-375$ & $345-378$ & 890 & 12 \\
\hline
\end{tabular}

a Concentrations are for oscillator-preamplifer stage. Amplifier stage concentrations are $1 / 3$ listed values.

b See Figure 3 for unusual tuning curve.

\section{Determination of Optimum Dye Ratio}

In an experiment where the dye laser was initially filled with PTP at its concentration optimum (oscillator-preamplifier stage) of $1.5 \times 10^{-3} \mathrm{M}(2)$, the growth of the mixture lasing was monitored as small volumes of concentrated DMQ solution were added. Typical tuning curves for these mixtures are shown in Figures $1 \mathrm{~b}$ and 1c. There are several noteworthy characteristics of these dye mixture tuning curves. First, as the dye DMQ is added, PTP lasing is attenuated overall and the tuning curve is augmented with a new peak at $356 \mathrm{~nm}$. Second, at $350 \mathrm{~nm}$, where neither dye lases strongly, the mixture lases strongly, Third, while the mixture typically shows a lower conversion efficiency than pure dyes, the breadth of the resulting tuning curve, with a full tuning range of 337-371 nm, represents some $34 \mathrm{~nm}$ of useful range. Fourth, the two maxima of the mixture's tuning curve, at roughly 344 and $356 \mathrm{~nm}$, are comparable in intensity at the dye molar concentration ratio of $[\mathrm{PTP}] /[\mathrm{DMQ}]=3.5$. At this molar ratio, the concentration of each dye $\left([\mathrm{PTP}]=1.1 \times 10^{-3} \mathrm{M}\right.$, $\left.[D M Q]=0.31 \times 10^{-3} \mathrm{M}\right)$ is lower than the corresponding single dye concentration optimum (see Table 1 for relevant values).

\section{Determination of Optimum Total Dye Concentration}

A second experiment was done holding the molar concentration ratio of two dyes fixed at the value of 3.5 (near equal intensity for lasing at 344 and $356 \mathrm{~nm}$ ). A stock concentrated solution of the fixed-ratio dye mixture was added stepwise to circulator 


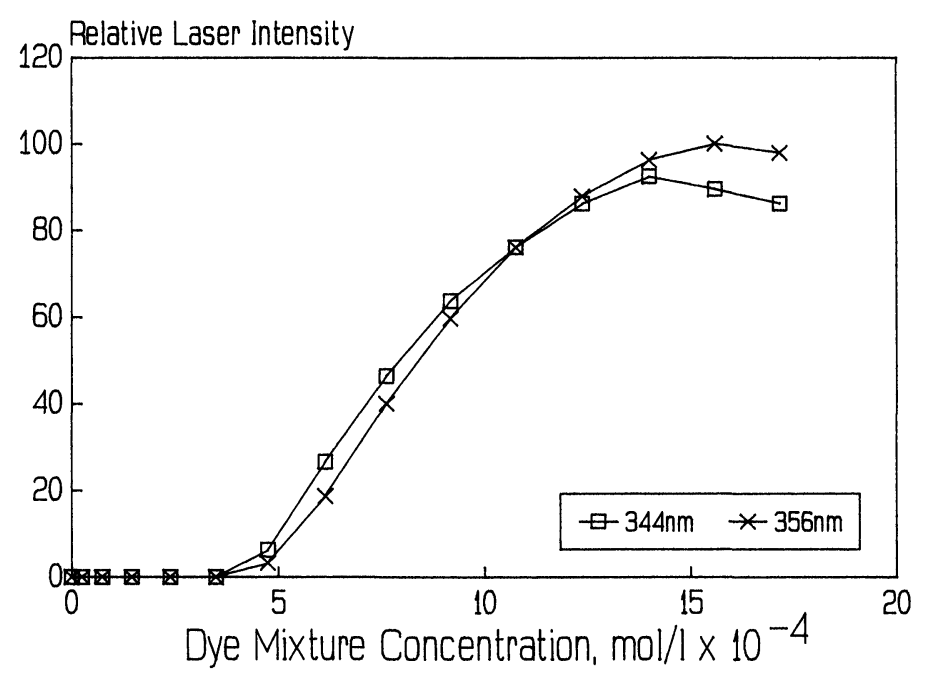

Figure 2 The relative laser intensity (pulse energy versus total dye concentration in the PTP/DMQ dye mixture with a fixed molar ratio of 3.5 .

solvent and the tuning curves recorded. The resulting concentration curves for the mixture are shown in Figure 2. Looking at the curves, it can be seen that the laser intensity ratio of the peaks at 344 and $356 \mathrm{~nm}$ remains roughly invariant throughout the concentration curve for the dye mixture. The optimum value of the total dye concentration (osc-preamp stage) is $1.4 \times 10^{-3} \mathrm{M} \quad\left([\mathrm{PTP}]=1.1 \times 10^{-3} \mathrm{M}\right.$; $\left.[D M Q]=0.31 \times 10^{-3} \mathrm{M}\right]$ based on an assumed need for equal intensity of the tuning curve's two features. In an approximate sense, the mixture at a molar ratio of 3.5 acts as a single hybrid ultraviolet dye with a $34 \mathrm{~nm}$ tuning range.

\section{Dye Mixture Lifetime}

The mixture of PTP and DMQ is also robust, that is, the dye mixture half-life is relatively long and comparable to, or exceeds, that of the dyes individually (Table 1). In addition, the dye mixture degrades symmetrically, preserving the near equal intensities of the 344 and $356 \mathrm{~nm}$ peaks. Simply put, attenuation of the dye concentration through photochemical degradation does not diminish the mixture's tunability.

\section{The TTPTP/PQP Mixture}

The dyes TTPTP (Exalite 348) and PQP represent stable terphenyl and quaterphenyl laser dyes when used in $p$-dioxane. In the former case, the new dye TTPTP (2) lases efficiently and has a half-life comparable to PQP (see Table 1). These dyes are described individually in Figure 3a where the tuning curves are compared. PQP shows two vibronic components (at 352 and $368 \mathrm{~nm}$ ) which are clearly separated in its tuning curve. In fact, the lack of intensity at $357 \mathrm{~nm}$ in the PQP tuning curve limits the 


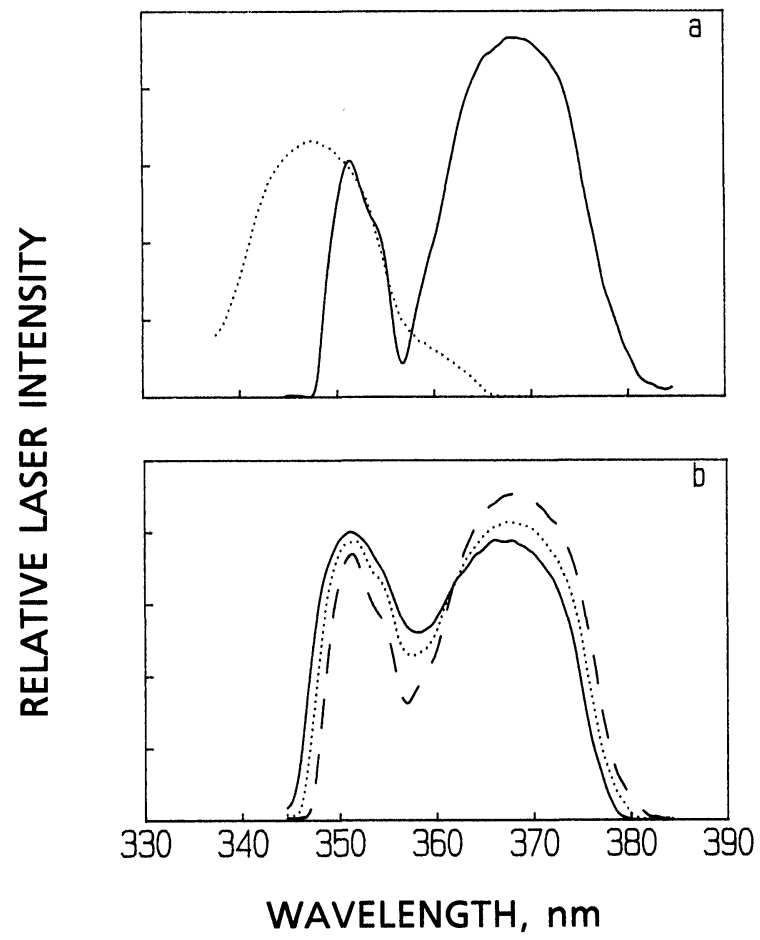

Figure 3 The tuning curves for the TTPTP/PQP dye mixture. All curves are correctly scaled vertically with respect to each other. Panel 3a: TTPTP only, dotted line; PQP only, solid line. Panel 3b: dashed line, $\left[\right.$ TTPTP] $=2.2 \times 10^{-4} \mathrm{M}, \quad[\mathrm{PQP}]=4.2 \times 10^{-4} \mathrm{M} ;$ dotted line, [TTPTP] $=4.6 \times 10^{-4} \mathrm{M}$, $[\mathrm{PQP}]=3.8 \times 10^{-4} \mathrm{M}$; solid line, $[\mathrm{TTPTP}]=6.8 \times 10^{-4} \mathrm{M},[\mathrm{PQP}]=3.5 \times 10^{-4} \mathrm{M}$.

usefulness of the dye near this wavelength. It should be noted that the lasing of TTPTP at $348 \mathrm{~nm}$ occurs predominantly in the region of the first, weaker, vibronic component of the PQP gain curve at $352 \mathrm{~nm}$. Thus, in this situation TTPTP can directly augment the weak PQP lasing. Experiments similar to those described above were done for this mixture and the results are briefly summarized below.

The binary dye mixture of TTPTP and PQP offers an excellent dye mixture over the region 345-380 $\mathrm{nm}$. Figure $3 \mathrm{~b}$ shows tuning curves at several stages of the addition of the dye TTPTP into the dye PQP. As in the case of the dye pair PTP/DMQ, the present mixture shows tuning curves with individual peaks which are relatively unaffected by the total concentration of the dye mixture. That is, as long as the molar ratio of TTPTP to PQP $([\mathrm{TTPTP}] /[\mathrm{PQP}]=1.9)$ is maintained, the tuning curve shows the desired broadness and near equal intensity of lasing at 352 and 367 $\mathrm{nm}$.

The TTPTP/PQP dye mixture is also robust and shows a half life comparable to each of its components (see Table 1). Photodegradation of the mixture through use results in a nearly symmetrical loss of the two dyes, thus maintaining a broad tuning curve at all stages of practical use. 


\section{ANALYSIS AND FINAL COMMENTS}

The terms "booster" dye and "active" dye have been introduced by Pavlopoulos (4) in a discussion of visible region laser dye mixtures. That notation is also useful in the present cases even though the degree of energy transfer for our dye mixtures is certainly less than that observed by Pavlopoulos. In this notation, the dyes PTP and TTPTP would be labelled "booster" dyes; PQP and DMQ "active" dyes. All of the laser dyes in this study are of the $\mathrm{A}_{2}$ a configuration (5) with only slight shifts in their absorption and fluorescence maxima. Thus, there is minor overlap of the fluorescence of the "booster" dye (shorter wavelength emitter) with the red edge of the absorption of the "active" dye (longer wavelength emitter). In spite of this lack of strong overlap, these binary mixtures function very effectively.

In the oscillator-preamplifier-amplifier dye laser configuration used in these studies, the output beam is determined in the first $2 \mathrm{~mm}$ of the transversely pumped dye flow cells (3). In a simple model one might think of the booster dye in this case as dumping its fluorescence photons into the laser cavity to boost the active dye's region of low optical gain. The partitioning of $308 \mathrm{~nm}$ excitation energy between the two dyes in the first $2 \mathrm{~mm}$ of dye cell depth was calculated for all the mixtures formed. The fluorescence spectra of the individual dyes (determined at equal concentrations) were then scaled by a partition ratio (ratio of the absorbances of each dye in the first 2 $\mathrm{mm}$ of path) at $308 \mathrm{~nm}$. Appropriately adding the weighted fluorescence spectra of the two dyes in a mixture gives a composite spectrum, the top portion of which closely resembles the associated mixture tuning curve. This is indicated in Figure $1 b$ and 1c for the PTP/DMQ mixture. A similar set of curves was constructed for the TTPTP/PQP mixture and very similar results were found. Allowing for a relatively high threshold of optical gain, the computed mixture fluorescences, specifically the top portions of the curves, closely resemble the associated tuning curves.

The close correspondence between the tuning curves and the fluorescence spectra of the mixtures is interpreted to mean that the principal mechanism for the lasing of the dye mixtures is simple. Namely, each dye independently contributes photons to the laser cavity and both dyes lase in concert. Indeed, we find that intermolecular energy transfer plays no strong role in the explanation of the dye mixture tuning curves.

In summary, two mixtures of ultraviolet dyes in $p$-dioxane have been described. These two mixtures are robust and degrade symmetrically producing broad tuning curves throughout the normal use of the mixture. A simple model of the lasing of the two mixtures based on independent fluorescences of the dyes seems to semiquantitatively describe the results obtained. On this basis, one should be able to easily extend these results to other useful laser dye mixtures by using the available absorption spectra, tuning curves and the fluorescence spectra of the separate dyes.

\section{Acknowledgements}

The authors thank Dr. Richard N. Steppel, Exciton, Inc., for his valuable suggestions concerning dye mixtures and Elaine M. Seliskar for her assistance with the manuscript. This work was supported, in part, 
by a grant from the Thomas Edison Fund of the State of Ohio, and by Academic Challenge Funds of the State of Ohio awarded to the Chemistry Department of the University of Cincinnati.

\section{References}

1. See, for example, K. H. Drexhage, "Structure and properties of laser dyes", in Topics in Applied Physics, Vol. 1, Dye Lasers, Springer-Verlag, New York (1990), pp 155-200.

2. D. J. Schneider, D. A. Landis, P. A. Fleitz, C. J. Seliskar, J. M. Kauffman, and R. N. Steppel, Laser Chemistry 11, 49-62 (1991).

3. Lambda Physik FL3002E dye laser manual.

4. T. G. Pavlopoulos, Opt. Comm. 24, 170-174 (1978); 38, 299-302 (1981)

5. T. G. Pavlopoulos and P. R. Hammond, J. Amer. Chem. Soc. 96, 6568-6579 (1974). 